\title{
Banxia xiexin decoction affects drug sensitivity in gastric cancer cells by regulating MGMT expression via IL-6/JAK/STAT3-mediated PD-L1 activity
}

\author{
XUAN FENG ${ }^{1,2}$, FENG XUE $^{3}$, GUIHUA HE ${ }^{4}$, QING NI $^{1,3}$ and SUIPING HUANG ${ }^{4}$ \\ ${ }^{1}$ Institute of Translational Medicine, Medical College, Yangzhou University; ${ }^{2}$ Jiangsu Key Laboratory of \\ Integrated Traditional Chinese and Western Medicine for Prevention and Treatment of Senile Diseases, \\ Yangzhou, Jiangsu 225001; ${ }^{3}$ Department of Surgery, Affiliated Hospital of Yangzhou University, \\ Yangzhou, Jiangsu 225000; ${ }^{4}$ Department of Gastroenterology, The Second Affiliated Hospital of \\ Guangzhou University of Chinese Medicine, Guangzhou, Guangdong 510120, P.R. China
}

Received September 23, 2020; Accepted January 15, 2021

DOI: $10.3892 / \mathrm{ijmm} .2021 .4998$

\begin{abstract}
Banxia xiexin decoction (BXXX) is a classic preparation used to treat gastrointestinal diseases, and also has certain therapeutic effects on gastrointestinal tumors. BXXX has been reported to regulate the expression of proteins associated with drug resistance and sensitivity in tumors, and thus, the aim of the present study was to investigate the mechanisms of BXXX drug sensitivity in gastric cancer (GC). The expression levels of programmed cell death 1 ligand 1 (PD-L1), 6-O-methylguanine-DNA methyltransferase (MGMT) and STAT3 were immunohistochemically detected in the cancer and adjacent non-cancer tissues of patients with GC, and in vitro experimentation was conducted using drug-resistant and -sensitive GC cells. The expression levels of PD-L1, MGMT and STAT3 were determined using reverse transcription-quantitative PCR. Different concentrations of BXXX drug serum were used to treat the cells and the cellular inhibition rate was assessed using a Cell Counting Kit-8 assay. Flow cytometry was used to detect apoptosis, and western blot analysis was used to detect the expression levels of IL-6, IFN- $\gamma$, JAK/STAT3 pathway proteins, PD-L1 and MGMT. The association between PD-L1 and MGMT protein expression levels was subsequently assessed via
\end{abstract}

Correspondence to: $\mathrm{Dr}$ Qing $\mathrm{Ni}$, Institute of Translational Medicine, Medical College, Yangzhou University, 188 Hanjiang Middle Road, Yangzhou, Jiangsu 225001, P.R. China

E-mail: nqingnn@163.com

Professor Suiping Huang, Department of Gastroenterology, The Second Affiliated Hospital of Guangzhou University of Chinese Medicine, 111 Dade Road, Guangzhou, Guangdong 510120, P.R. China E-mail: gzdoctorhsp@163.com

Key words: banxia xiexin decoction, drug sensitivity, gastric cancer, 6-O-methylguanine-DNA methyltransferase, programmed cell death 1 ligand 1, IL-6/JAK/STAT3 co-immunoprecipitation. Furthermore, in vivo studies were conducted following the establishment of a drug-resistant tumor-bearing mouse model, where GC tumor size was assessed under different treatment conditions, and western blot analysis was used to detect the expression of related pathway proteins. The expression levels of PD-L1, MGMT and STAT3 were significantly increased in GC tissues, GC cells and cisplatin-resistant cells. Furthermore, BXXX inhibited the proliferation of drug-resistant cells and promoted the inhibitory effects of chemotherapeutic drugs on drug-resistant cells. BXXX also inhibited the expression levels of IL-6, IFN- $\gamma$ and JAK/STAT3 pathway proteins, as well as the expression levels of PD-L1 and MGMT. Colivelin, an activator of STAT3, reversed the effects of BXXX on drug-resistant GC cells, and significantly reversed the effect of BXXX on PD-L1 expression. In conclusion, BXXX was found to influence the drug sensitivity of GC cells by regulating the expression of MGMT. This process functions viaPD-L1, which was itself mediated by IL-6/JAK/STAT3 signaling.

\section{Introduction}

Gastric cancer (GC) is one of the most common malignant tumor types of the digestive system and is the second leading cause of cancer-associated mortality worldwide (1). GC morbidity and mortality rates remain high. By 2018, the global mortality rate of gastric cancer had reached $8.2 \%$ (2). At present, chemotherapy is still an important treatment option for patients with advanced disease, of which cisplatin (DDP) is commonly used (3). However, long-term use often results in drug tolerance, which is an important issue that requires urgent attention (4). Due to its unique ability to inhibit tumor growth, improve patient quality of life and prolong survival time, Traditional Chinese Medicine (TCM) has become a valuable research hotspot and focus in the field of tumor adjuvant therapy and drug sensitivity $(5,6)$.

Banxia xiexin decoction (BXXX) is a classic TCM prescription used to treat gastrointestinal diseases. Previous studies have reported that BXXX effectively treats digestive tumors, as well 
as those of other bodily systems (7). For example, BXXX can alleviate colon cancer (8), lung cancer (9), gallbladder cancer (10) and cancer symptoms. In addition, serum containing BXXX was found to inhibit the proliferative, invasive and metastatic abilities of GC GC9811-P cells, thereby blocking the peritoneal metastasis of GC (11). BXXX also regulates the expression of STAT3 proteins in gastric adenocarcinoma and gastric mucosal epithelial cells (12). In inflammatory diseases and GC, BXXX can regulate the levels of inflammatory factors, including IFN- $\gamma$ and IL-6 (13). In our previous study, the BATMAN-TCM bioinformatics analysis tool (http://bionet.ncpsb.org/batman-tcm/) was used to analyze drug composition and target prediction, and BXXX was found to also exert its effects on a variety of proteins associated with tumor drug resistance and sensitivity, such as 6-O-methylguanine-DNA methyltransferase (MGMT) (data not yet published). Moreover, MGMT is strongly associated with the occurrence and development of GC, especially drug resistance (14).

Programmed cell death protein 1 (PD-1) and programmed cell death 1 ligand 1 (PD-L1) remain a research hotspot for drug resistance in tumors, but the associated resistance mechanisms are more closely associated with those of EGFR-TKI and tumor immune escape (15-18). However, in addition to its ability to influence T cells via its ligand PD-1, PD-L1 can also impact tumor cells. For example, PD-L1 was found to increase the expression levels of various multi-drug resistance proteins in tumor cells. Moreover, the expression of PD-L1 is increased following tumor cell DNA damage, which is also closely associated with the activation of DNA repair mechanisms $(19,20)$.

IL-6 and IFN- $\gamma$ regulate the JAK/STAT3 pathway to induce the expression of PD-L1 in tumor-related macrophages, thus regulating the occurrence and development of lung cancer (21). Moreover, the activator protein-2 (AP-2) factor inhibited the IL-6/JAK2/STAT3 signaling pathway, decreasing the expression levels of MGMT and PD-L1 in glioma tissues and cell lines (22). Therefore, it was hypothesized that BXXX may also moderate PD-L1 expression by regulating IL-6, IFN $\gamma$ and the downstream STAT3 pathway, and potentially influence the expression of MGMT.

Thus, in the present study, the mechanism of drug sensitivity of BXXX to GC was investigated via animal experiments and cell experiments.

\section{Materials and methods}

BXXX preparation. BXXX (Pinellia ternata, $15 \mathrm{~g}$; Scutellaria baicalensis Georgi, 9 g; dried ginger, $9 \mathrm{~g}$; ginseng, $9 \mathrm{~g}$; honey-fried licorice root, $9 \mathrm{~g}$; Coptis chinensis, $3 \mathrm{~g}$; and jujube, 4 pieces) was provided by The Affiliated Hospital of Yangzhou University (Yangzhou, China). BXXX was processed into granules by The Second Affiliated Hospital of Guangzhou University of Chinese Medicine (Guangzhou, China). All herbs were obtained from the original sources and met the standards listed in the National Pharmacopoeia of China, and were identified by Guangdong Provincial Hospital of Chinese Medicine. The decoction was made by boiling the mixture twice in distilled water at $100^{\circ} \mathrm{C}$ for $30 \mathrm{~min}$. The drug solution was then cooled and dried into granules. Finally, the granule was dissolved in $0.5 \%$ sodium carboxymethyl cellulose. The present study did not use High Performance Liquid
Chromatography to determine the active components, because $\mathrm{BXXX}$ is a classic prescription, and its active components have been determined in the study of Chen et al (23).

Clinical specimens. Clinicopathological data, GC tissue and adjacent tissue samples $(\sim 1 \mathrm{~cm}$ from the cancer tissue) were obtained from 10 patients ( 5 women, 5 men, aged between 37-65 years) with GC who were admitted to The Affiliated Hospital of Yangzhou University between June 2019 and December 2019. None of the patients had received adjuvant radiotherapy or chemotherapy prior to surgery. All samples were verified by experienced pathologists and immediately stored in liquid nitrogen $\left(-80^{\circ} \mathrm{C}\right)$. The present study was approved by The Affiliated Hospital of Yangzhou University, and each patient provided written informed consent.

Cell culture. Normal gastric mucosa epithelial and GC cell lines (AGS, SNU-1, KATO III and NCI-N87) were purchased from the Type Culture Collection of the Chinese Academy of Science, and incubated in DMEM supplemented with $10 \%$ FBS (both Gibco; Thermo Fisher Scientific) at $37^{\circ} \mathrm{C}\left(5 \% \mathrm{CO}_{2}\right)$. The human DDP-resistant gastric adenocarcinoma cell lines AGS/DDP and NCI-N87/DDP were purchased from Gefan Biotechnology (Shanghai, China). In order to maintain drug resistance in AGS/DDP and NCI-N87/DDP cells, the culture media were supplemented with DDP (cat. no. B00798003; Best reagent; www.best-reagent.com) at a final concentration of $2 \mathrm{nmol} / \mathrm{l}$. AGS/DDP cells were pre-treated with colivelin (50 $\mu \mathrm{g} / \mathrm{ml}$; Tocris Biosciences) for $2 \mathrm{~h}$ at $37^{\circ} \mathrm{C}$, a STAT3 activator, and the cells were divided into AGS/DDP, 15\% BXXX, $15 \%$ BXXX + covlivelin, 15\% BXXX + DDP and 15\% BXXX + DDP + covlivelin groups.

Preparation of a GC xenograft model in nude mice. A total of 20 healthy female BALB/C nude mice (age, 6-8 weeks; weight, 16-20 g) acquired from The Affiliated Hospital of Yangzhou University were kept in an environment with a constant temperature of $25^{\circ} \mathrm{C}$ and humidity of $30-70 \%$ with $12 \mathrm{~h}$ light/dark cycle. The mice were fed normally and maintained in specific pathogen-free conditions. All animal procedures and experimental methods were approved by the Committee on the Ethics of Animal Experiments of The Affiliated Hospital of Yangzhou University, and were conducted in accordance with the ARRIVE guidelines (24). AGS/DDP cells were used to establish a drug-resistant tumor-bearing mouse model of GC. The mice were subcutaneously inoculated with AGS/DDP cells $(150 \mu \mathrm{l})$ in the logarithmic growth stage $\left(6 \times 10^{7} \mathrm{U} / \mathrm{ml}\right)$ near the back-right upper limb. Tumors were visible after 3-5 days, at which point $\mathrm{BXXX}(9 \mathrm{~g} / \mathrm{kg})$ was administered daily by gavage and DDP $(5 \mathrm{mg} / \mathrm{kg}$, intraperitoneal injection, once a week). The body weight and tumor volume of each mouse were recorded for 5 weeks, and the maximum tumor volume was $1,100 \mathrm{~mm}^{3}$. Model mice were treated with DDP, BXXX and a combination of both DDP and BXXX, and then divided into the AGS/DDP, DDP, BXXX and DDP + BXXX groups. Each group comprised of five mice. After successful modeling, the mice were sacrificed by cervical dislocation (the mice stopped breathing, their hearts stopped beating, their muscles relaxed, their body temperature dropped and they did not respond to external stimuli) and the tumor tissues were removed for 
subsequent experimentation. In addition, $3 \mathrm{ml}$ serum each rats containing BXXX was prepared at concentrations of 5, 10 and $15 \%$, and used in cell experiments.

Data. The STRING website (version 11.0; https://string-db. org/) was used to predict the relationship between IL-6, JAK/STAT3 and MGMT.

Immunohistochemistry (IHC). GC and para-carcinoma tissue (thickness, $\sim 4 \mu \mathrm{m}$ ) were fixed with $4 \%(\mathrm{w} / \mathrm{v})$ paraformaldehyde at $4^{\circ} \mathrm{C}$ for $24 \mathrm{~h}$, deparaffinized and embedded in paraffin once more. The tissues were incubated with primary antibodies against PD-L1 (1:100; cat. no. sc-293425; Santa Cruz Biotechnology, Inc.), MGMT (1:100; cat. no. sc-166528; Santa Cruz Biotechnology, Inc.) and STAT3 (1:100; cat. no. sc-8019; Santa Cruz Biotechnology, Inc.) overnight at $4^{\circ} \mathrm{C}$, and then with secondary antibodies (1:5,000; Abcam) for $1 \mathrm{~h}$ at $37^{\circ} \mathrm{C}$. Subsequently, 3,3'-diaminobenzidine tetrahydrochloride staining was conducted (Sigma-Aldrich; Merck KGaA) at room temperature for $5 \mathrm{~min}$. The results were observed under a light contrast microscope (BX51; Olympus Corporation; magnification, $\mathrm{x} 200$ ).

Reverse transcription-quantitative $(R T-q) P C R$. Total RNA was extracted from the cells using TRIzol ${ }^{\circledR}$ reagent (Invitrogen; Thermo Fisher Scientific, Inc.) according to the manufacturer's instructions. The RNA was reverse transcribed into complementary DNA using the First-Strand cDNA Synthesis kit, and qPCR was conducted using the SuperScript III Platinum SYBR-Green One-Step qRT-PCR kit (both Invitrogen; Thermo Fisher Scientific, Inc.) according to the manufacturer's instructions. The thermocycling conditions were as follows: Initial denaturation at $95^{\circ} \mathrm{C}$ for $10 \mathrm{~min}$, followed by 40 cycles of $95^{\circ} \mathrm{C}$ for $10 \mathrm{sec}, 55^{\circ} \mathrm{C}$ for $10 \mathrm{sec}$ and $72^{\circ} \mathrm{C}$ for $30 \mathrm{sec}$. Relative expression levels were calculated using the $2^{-\Delta \Delta \mathrm{Cq}}$ method (25). The following primers were used: PD-L1 forward, 5'-TTT GCTGAACGCCCCATA-3' and reverse, 5'-TGCTTGTCC AGATGACTTCG-3'; MGMT forward, 5'-GCGTTTCGA CGTTCGTAGGT-3' and reverse, 5'-CACTCTTCCGAAAAC GAAACG-3'; STAT3 forward, 5'-CTCAACTTCAGACCC GTCAACA-3' and reverse, 5'-GCTCCACGATTCTCTCCT CCA-3'; and GAPDH forward, 5'-CTCGCTTCGGCAGCA CA-3' and reverse, 5'-AACGCTTCACGAATTTGCGT-3'.

Cell Counting Kit 8 (CCK-8) assay. Cells were cultured in a 96 -well plate $\left(5 \times 10^{3}\right.$ cells/well). Following treatment, $10 \mu \mathrm{l}$ CCK-8 solution (Dojindo Molecular Technologies, Inc.) was added to each well and the cells were incubated at $37^{\circ} \mathrm{C}$ for $4 \mathrm{~h}$, according to the manufacturer's instructions. Finally, the absorbance was measured at $450 \mathrm{~nm}$ using a microplate reader (Bio-Rad Laboratories, Inc.).

Flow cytometry. Following the appropriate treatment, the cells were harvested for apoptosis analyses. Apoptosis was assessed using the FITC-Annexin V Apoptosis Detection kit II (BD Biosciences) according to the manufacturer's instructions, and flow cytometry was performed using the BD FACSCanto II Flow Cytometer (BD Biosciences). The data were analyzed using FlowJo software (version 1.46; FlowJo LLC).
Western blotting. Cells were lysed with RIPA lysis buffer (Beyotime Institute of Biotechnology) and incubated on ice for $30 \mathrm{~min}$. The protein concentrations were determined using a BCA protein assay kit (Bio-Rad Laboratories, Inc.). A total of $40 \mu \mathrm{g}$ protein/well was separated using $10 \%$ SDS-PAGE gels, and subsequently transferred to PVDF membranes. The membranes were blocked with $10 \%$ skimmed milk for $2 \mathrm{~h}$ at room temperature, followed by incubation with primary antibodies overnight at $4^{\circ} \mathrm{C}$. The membranes were subsequently incubated with goat anti-rabbit HRP-conjugated IgG secondary antibodies (1:5,000; cat. no. ab96899; Abcam) at room temperature for $1 \mathrm{~h}$. The signals were detected using an enhanced chemiluminescence reagent (Cytiva) and ImageJ software (version 146; National Institutes of Health) was used to semi-quantify the fold-changes in protein expression. The following primary antibodies were used in the present study: Anti-IL-6 (1:1,000; cat. no. 12912s), anti-JAK1 (1:1,000; cat. no. 29261s), anti-STAT3 (1:1,000; cat. no. 9139s), anti-IFN receptor (IFNR; 1:1,000; cat. no. 8455s), anti-PD-L1 (1:1,000; cat. no. 13684s), anti-MGMT (1:1,000; cat. no. 86039s) and anti-GAPDH (1:1,000; cat. no. 5174S). All antibodies were obtained from Cell Signaling Technology, Inc.

Co-immunoprecipitation (co-IP). Cells were harvested 24 or $48 \mathrm{~h}$ post-transfection, and $8 \mathrm{ml}$ lysis buffer (containing protease inhibitor; Beyotime Institute of Biotechnology) was added to each sample. The cells were lysed on ice $\left(4^{\circ} \mathrm{C}\right)$ for $30 \mathrm{~min}$, after which the supernatants were harvested by centrifugation for $30 \mathrm{~min}$ at $300 \mathrm{xg}$ at $4^{\circ} \mathrm{C}$. A small amount of each lysate was retained for western blot analysis, and the remainder was added to the cell lysate containing $1 \mu \mathrm{g}$ of the anti-PD-L1 (1:1,000; cat. no. 13684s) and anti-MGMT (1:1,000; cat. no. 86039s). The samples were incubated overnight at $4^{\circ} \mathrm{C}$. Next, $10 \mu \mathrm{l}$ protein A agarose beads (washed with lysate; Sigma-Aldrich; Merck KGaA) were added to the aforementioned cell lysates, and slowly shaken at $4^{\circ} \mathrm{C}$ for $2-4 \mathrm{~h}$ to encourage antibody/bead coupling. Following immunoprecipitation, the coupled samples were centrifuged at $350 \times \mathrm{g}\left(4^{\circ} \mathrm{C}\right)$ for $30 \mathrm{~min}$, and the supernatant was discarded. The samples were then washed 3-4 times with $1 \mathrm{ml}$ buffer solution. Finally, $15 \mu \mathrm{l}$ SDS (2X) was added, and the samples were boiled for $5 \mathrm{~min}$ prior to western blot analysis.

Statistical analysis. SPSS version 21.0 (IBM Corp) was used to perform all statistical analyses, and the data are presented as the mean \pm standard deviation. Comparisons were made using one-way ANOVA followed by Tukey's post hoc test. $\mathrm{P}<0.05$ was considered to indicate a statistically significant difference. The data of IHC were presented as the median \pm interquartile range and analyzed using a non-parametric test (Wilcoxon signed-rank test). Each experiment was performed $\geq 3$ times.

\section{Results}

Expression levels of PD-L1, MGMT and STAT3 in drug-resistant $G C$ cells. The baseline patient data are presented in Table I. Fig. 1 shows the protein pathway diagram analyzed by the STRING website. The IHC results revealed that the expression levels of PD-L1, MGMT and STAT3 in GC tissues were significantly higher compared with those in 
Table I. Baseline characteristics of patients with gastric cancer.

\begin{tabular}{cccccc}
\hline Sample & Sex & Age (years) & Drinking & Smoking & Family history of cancer (any type) \\
\hline 1 & Male & 38 & Yes & No & Yes \\
2 & Male & 57 & Yes & Yes & No \\
3 & Male & 35 & No & Yes & Yes \\
4 & Male & 65 & Yes & No & No \\
5 & Male & 54 & Yes & No & No \\
6 & Female & 51 & No & No & No \\
7 & Female & 42 & No & Yes & Yes \\
8 & Female & 58 & No & No & Yes \\
9 & Female & 46 & Yes & No & No \\
10 & Female & 37 & No & Yes & \\
\hline
\end{tabular}

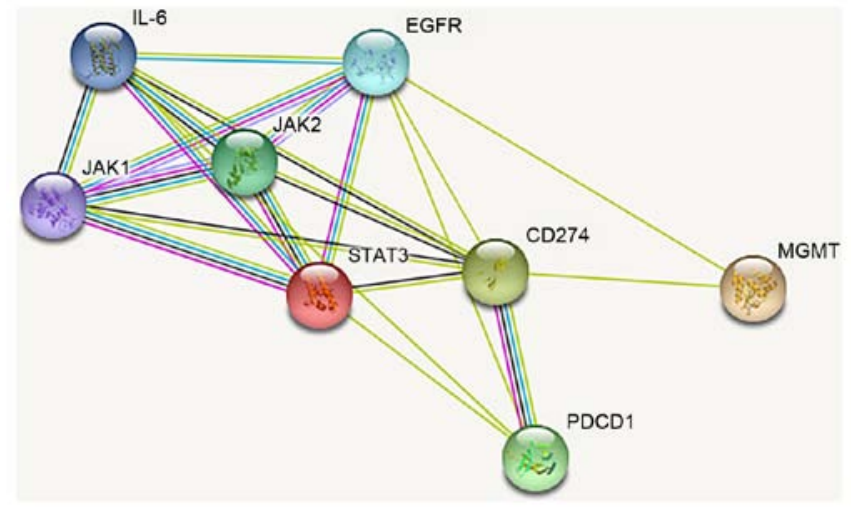

Figure 1. Protein pathway diagram analyzed by the STRING web site.

the para-carcinoma tissues (Fig. 2). Then, PD-L1, MGMT and STAT3 expression levels were detected in different GC cell lines. The results indicated that compared with those in GES1 cells, the expression levels of PD-L1, MGMT and STAT3 in GC cell lines were increased to varying degrees (Fig. 3A). Subsequently, two groups of AGS cells with significant increases in PD-L1, MGMT and STAT3 expression levels, as well as NCI-N87 cells (whose expression levels were not significantly increased) were selected for drug resistance induction, and designated as the GES1, AGS/DDP and NCI-N87/DDP groups. Compared with the GES1 group, the expression levels of PD-L1, MMT and STAT3 were also significantly increased (to a comparable degree) in the AGS/DDP and the NCI-N87/DDP groups (Fig. 3B), indicating that that PD-L1, MGMT and STAT3 are closely associated with drug resistance. Therefore, the inhibitory effects of DDP on AGS and NCI-N87 cells were also investigated. Compared with AGS and NCI-N87 cells, cellular proliferation was inhibited following drug resistance induction (Fig. 3C). As the PD-L1, MGMT and STAT3 expression levels were higher in AGS cells compared with in NCI-N87 cells, the DDP inhibition rate of the AGS cells was relatively low. Therefore, AGS and AGS/DDP cells were selected for further investigation.

$B X X X$ regulates the sensitivity of drug-resistant $G C$ cells. Serum samples containing different concentrations of BXXX were selected to assess its effects on the proliferation of AGS and AGS/DDP cells. As presented in Fig. 4A and B, AGS and AGS/DDP cell proliferation was inhibited at different concentrations of BXXX, and an increase in BXXX concentration resulted in a gradual increase in the inhibition rate. Thus, a concentration of $15 \%$ BXXX was used to further assess whether BXXX promoted the inhibitory effects of chemotherapeutic drugs on drug-resistant strains.

The CCK-8 assay results revealed that, compared with drug-sensitive AGS cells, AGS cell proliferation was inhibited after the addition of DDP, which was further retarded by $15 \%$ BXXX (Fig. 4C). The flow cytometric results demonstrated that, compared with the AGS group, the apoptotic rate of AGS/DDP cells was decreased. Compared with the AGS/DDP group, the apoptotic rate of the AGS/DDP + 15\% BXXX group was increased, and the rate of the AGS/DDP + DDP group was enhanced to a greater degree compared that of the AGS/DDP $+15 \%$ BXXX group. Moreover, compared with the AGS/DDP + DDP group, the apoptotic rate of the AGS/DDP + $15 \%$ BXXX + DDP group was further increased (Fig. 4D and E). The results indicated that BXXX could promote the inhibitory effect of chemotherapy drugs on drug-resistant cells.

The expression levels of key pathway proteins were determined. The expression levels of IL-6, INF- $\gamma$, JAK1, STAT3 and MGMT were significantly upregulated in the AGS/DDP group compared with the AGS group (Fig. 4F). Compared with the AGS/DDP group, IL-6, INF- $\gamma$, JAK1, STAT3 and MGMT expression levels were decreased in the AGS/DDP + DDP and AGS/DDP $+15 \%$ BXXX group, but this was more significant in the AGS/DDP + DDP group. The expression levels of IL-6, INF- $\gamma$, JAK1, STAT3 and MGMT were further decreased following DDP and 15\% BXXX co-treatment (Fig. 4F). These results demonstrated that $\mathrm{BXXX}$ and DDP exerted a greater inhibitory effect on the expression levels of pathway-related proteins when used synergistically.

$B X X X$ mediates drug-resistant $G C$ cell sensitivity by regulating the expression of MGMT via IL-6/JAK/STAT3-mediated $P D-L 1$. Using co-IP, PD-L1 was found to directly target and regulate MGMT (Fig. 5A and $\mathrm{B}$ ).

The CCK-8 results demonstrated that cell proliferation increased after the addition of Covlivelin, compared with $15 \%$ BXXX alone, as evident by the decrease in the inhibition rate. Compared with the $15 \% \mathrm{BXXX}+\mathrm{DDP}$ group, cell 

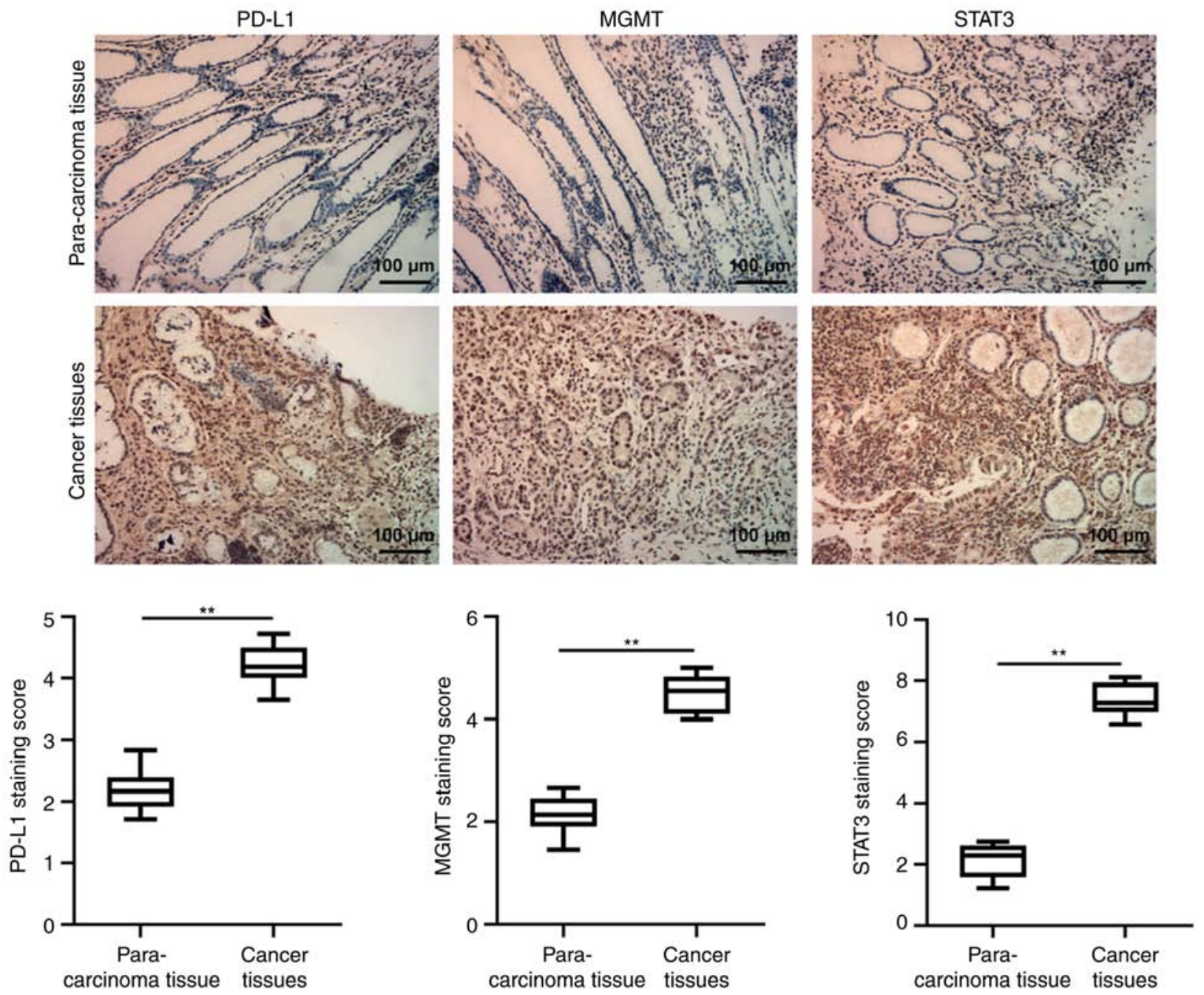

Figure 2. Immunohistochemistry was used to detect the expression levels of PD-L1, MGMT and STAT3 in para-carcinoma tissue and cancer tissues. Scale bar, $100 \mu \mathrm{m}$. Statistical analysis was conducted on the expression levels of PD-L1, MGMT and STAT3 in tissues. ${ }^{* *} \mathrm{P}<0.001$. PD-L1, programmed cell death 1 ligand 1; MGMT, 6-O-methylguanine-DNA methyltransferase.
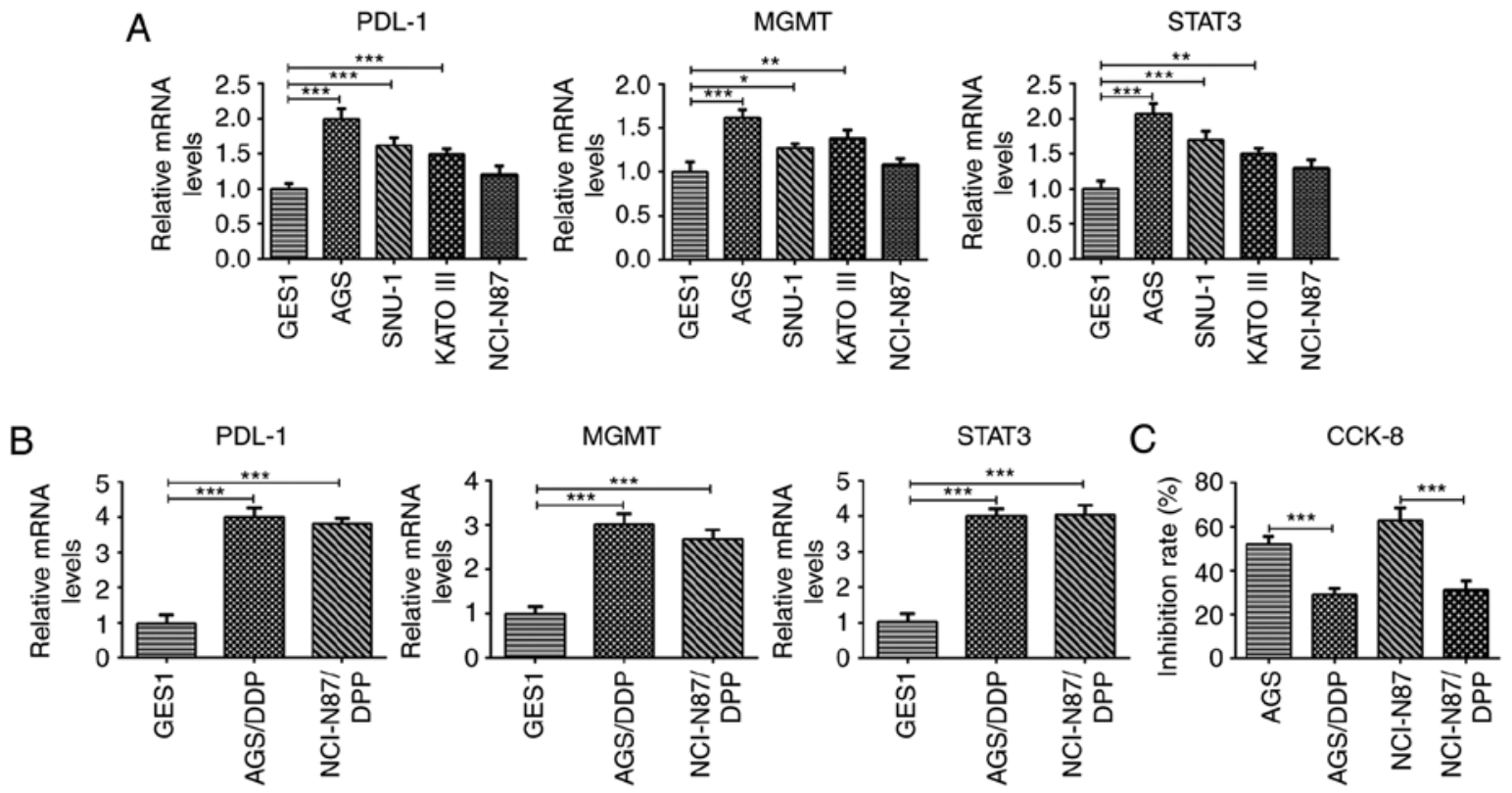

Figure 3. Expression levels of PD-L1, MGMT and STAT3. Reverse transcription-quantitative PCR were used to detect the expression levels of PD-L1, MGMT and STAT3 in (A) GC cell lines and (B) GC resistant cell lines AGS/DDP and NCI-N87/DDP. (C) A CCK-8 assay was used to assess the cell viability. ${ }^{*}<0.05$, ${ }^{* *} \mathrm{P}<0.01,{ }^{* * *} \mathrm{P}<0.001$. CCK, Cell Counting Kit; PD-L1, programmed cell death 1 ligand 1; MGMT, 6-O-methylguanine-DNA methyltransferase; DDP, cisplatin. 
A

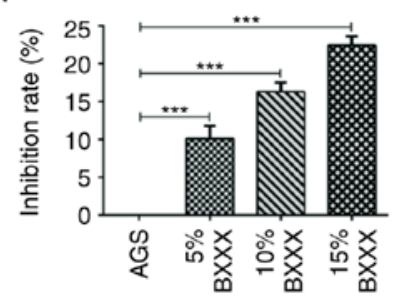

B

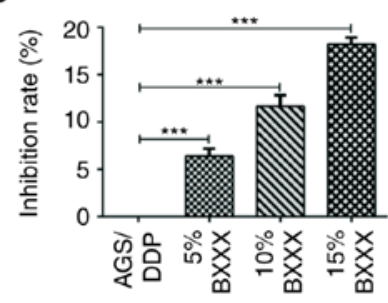

C

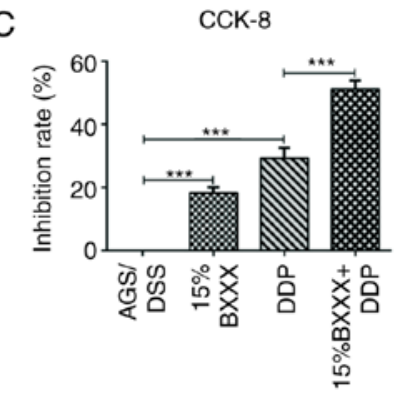

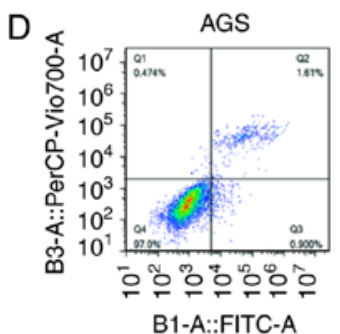

B1-A::FITC-A
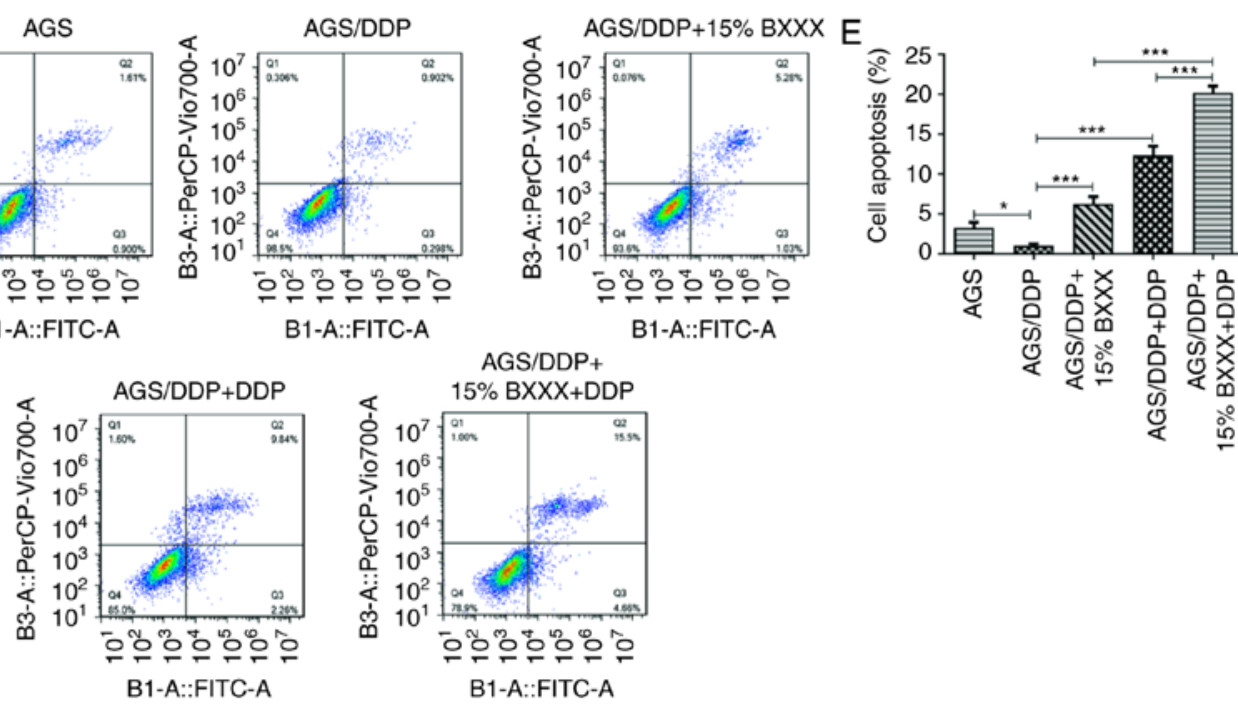

B1-A::FITC-A
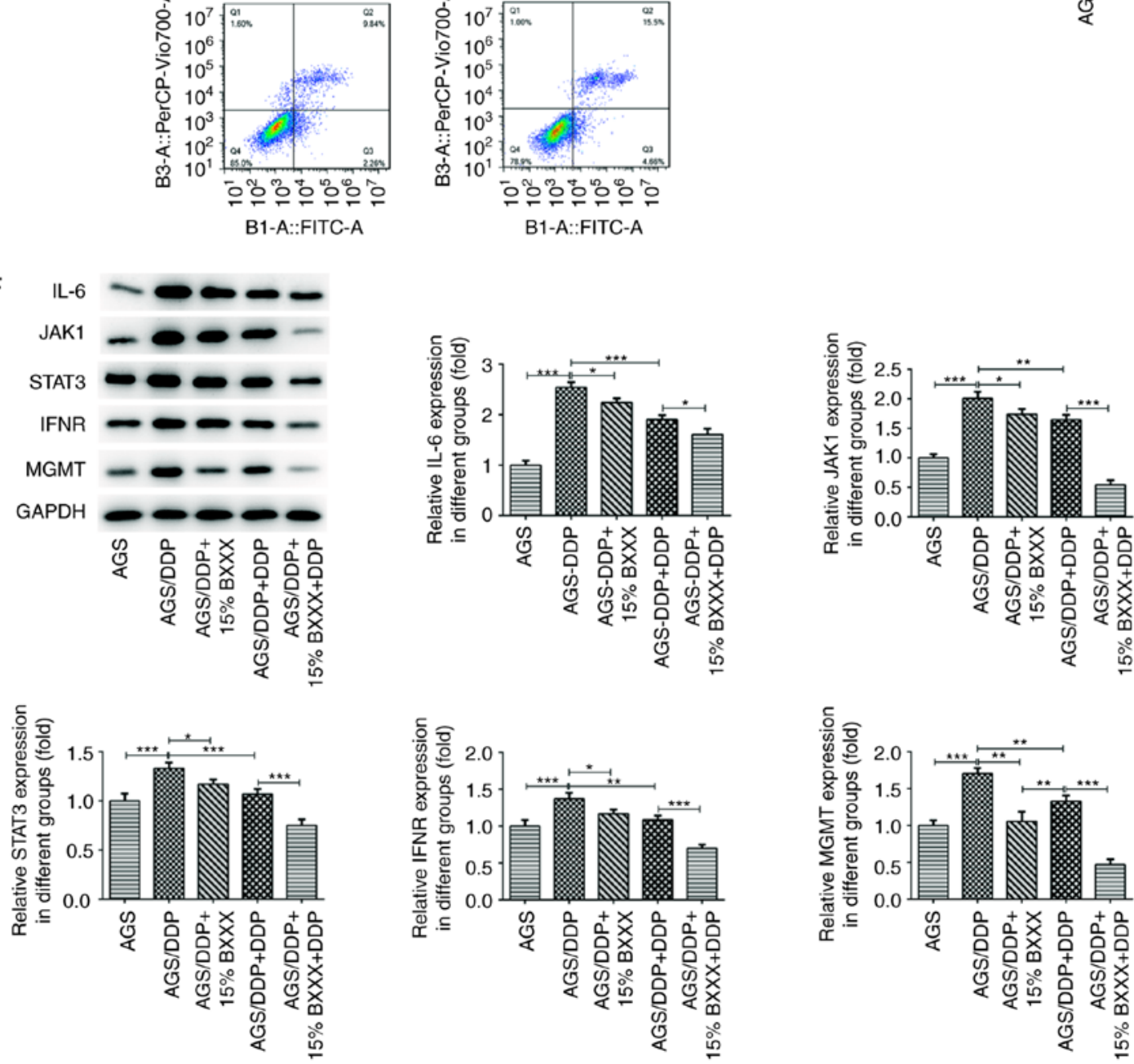

Figure 4. BXXX regulates the sensitivity of drug-resistant cells of GC. The CCK-8 assay detected the viability of (A) AGS cells and (B) AGS/DDP cells. (C) Cell viability after the addition of $15 \%$ BXXX and DDP, as determined with a CCK-8 assay. (D) Flow cytometry was used to detect cell apoptosis. (E) Statistical analysis of apoptotic cells. (F) Western blotting was used to measure the expression levels of the signaling pathway-related proteins IL-6, JAK1, STAT3, IFNR and MGMT. ${ }^{*} \mathrm{P}<0.05,{ }^{* *} \mathrm{P}<0.01,{ }^{* * *} \mathrm{P}<0.001$. CCK, Cell Counting Kit; PD-L1, programmed cell death 1 ligand 1; MGMT, 6-O-methylguanine-DNA methyltransferase; DDP, cisplatin; BXXX, Banxia xiexin decoction; IFNR, IFN receptor.

proliferation was enhanced following Covlivelin treatment (Fig. 6A). Flow cytometry was used to detect apoptosis, and the trend was consistent with that of the CCK- 8 results (Fig. 6B and C). The cellular expression levels of IL-6, INF- $\gamma$, 
A

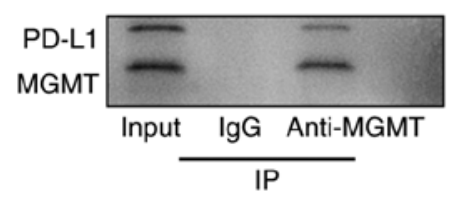

B

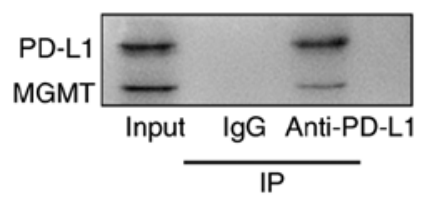

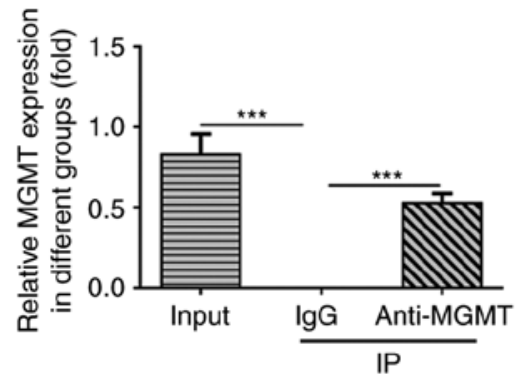
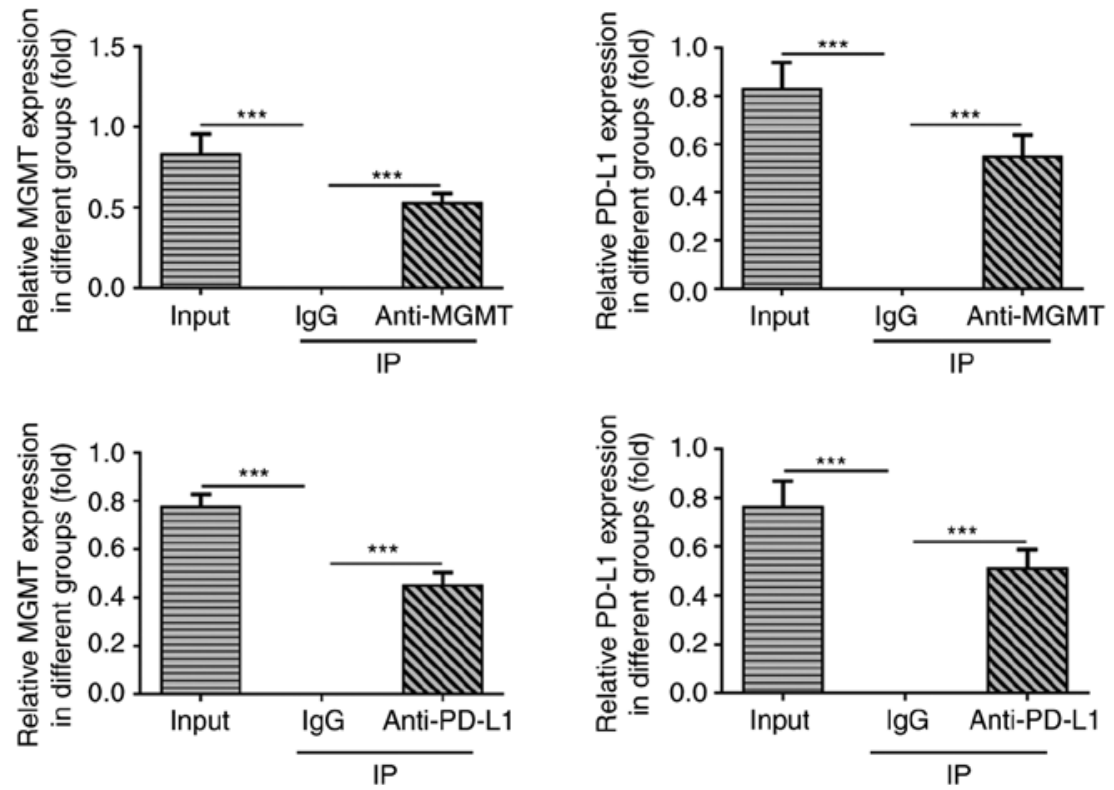

Figure 5. Association between PD-L1 and MGMT. Co-IP detected the relationship between PD-L1 and MGMT proteins. (A) Expression of PD-L1 and MGMT in cells after co-precipitation with MGMT. (B) Expression of PD-L1 and MGMT in cells after co-precipitation with PD-L1. *** P<0.001. PD-L1, programmed cell death 1 ligand 1; MGMT, 6-O-methylguanine-DNA methyltransferase; IP, immunoprecipitation.

JAK1, STAT3, PD-L1 and MGMT were then determined. The expression levels of IL-6, INF- $\gamma$, JAK1, STAT3, PD-L1 and MGMT in the $15 \%$ BXXX group showed an opposing trend to the expression levels in the $15 \% \mathrm{BXXX}+$ Covlivelin group, and PDL-1 expression was significantly increased following Covlivelin treatment (Fig. 6D). These findings indicated that following STAT3 activation, the tumor inhibitory effect of BXXX was not considerably pronounced, but that the effects of BXXX on DDP drug sensitivity were enhanced. In other words, BXXX has a direct impact on the pathways upstream of IL- 6 and INF- $\gamma$, as well as on MGMT, but has a less direct effect on PD-L1, which may be mediated by STAT3.

Tumor volume was decreased in the DDP and BXXX groups, compared with the AGS/DDP group. Moreover, compared with the DDP and BXXX groups, the tumor volume of the DDP + BXXX group was significantly decreased (Fig. 7A and $\mathrm{B})$. The expression levels of related pathway proteins were also detected in tumor tissues. As shown in Fig. 8, compared with the AGS/DDP group, IL-6, INF- $\gamma$, JAK1, STAT3 and MGMT expression levels were decreased in the DDP and BXXX group. The expression levels of IL-6, INF- $\gamma$, JAK1, STAT3 and MGMT were further decreased following DDP and BXXX co-treatment. In addition, the expression of PD-L1 did not change significantly in AGS/DDP and BXXX groups. Similarly, PD-L1 showed no significant change in DDP and $\mathrm{DDP}+\mathrm{BXXX}$. The expression levels of these proteins were consistent with those observed in vitro.

\section{Discussion}

TCM has unique antitumor features and can be used as an adjuvant treatment to compliment surgery, radiotherapy or chemotherapy (26). However, TCM can also serve a direct antitumor role. BXXX is a classic TCM preparation used for the treatment of gastrointestinal diseases, but which also exerts therapeutic effects against tumors (7). In the present study,
BXXX was found to promote the apoptosis of drug-resistant GC cells. When used in combination with DDP, the apoptotic rate was further increased compared with that of the DDP group alone, indicating that BXXX not only decreased the sensitivity of GC cells to DDP, but also inhibited GC cell proliferation.

The development of drug-resistant tumor cells is an important factor for chemotherapeutic efficacy, thus drug resistance in tumors is a current research focus. A previous study has demonstrated that the molecular mechanisms of tumor cell drug resistance involve multiple processes, such as alterations in drug metabolism, enhancing DNA damage repair and inhibiting apoptosis, but the specific mechanisms are still unknown (27). The expression of drug-resistance genes is an important factor affecting treatment susceptibility. MGMT is a DNA repair protein commonly expressed in human cells (encoded by the MGMT gene), and is currently recognized as a DNA repair enzyme associated with tumor cell resistance to alkalizing agents (28). Li et al (29) reported that MGMT was a prognostic and chemotherapy-sensitivity marker in GC. Moreover, the inhibition of MGMT-mediated autophagy decreases the chemotherapeutic sensitivity of GC cells to DDP (30). In the present study, MGMT expression was found to be significantly increased in GC tissues and cells, as well as in drug-resistant GC cell lines, which was consistent with the results of previous publications. In addition, drug composition analysis and target prediction revealed that BXXX has a certain regulatory effect on MGMT, but the specific mechanisms have not yet been reported (data not yet published). Therefore, in the present study, the role of BXXX in GC cell drug resistance was investigated, and the underlying mechanisms discussed.

The current study demonstrated that BXXX blocks the JAK/STAT3 signaling pathway by downregulating the expression of IFN $\gamma$ and IL-6, thereby reducing the expression of PD-L1 in drug-resistant GC cells. Another study showed that inhibition of ataxia telangiectasia mutated protein reversed epithelial-mesenchymal transition and the condensing potential 

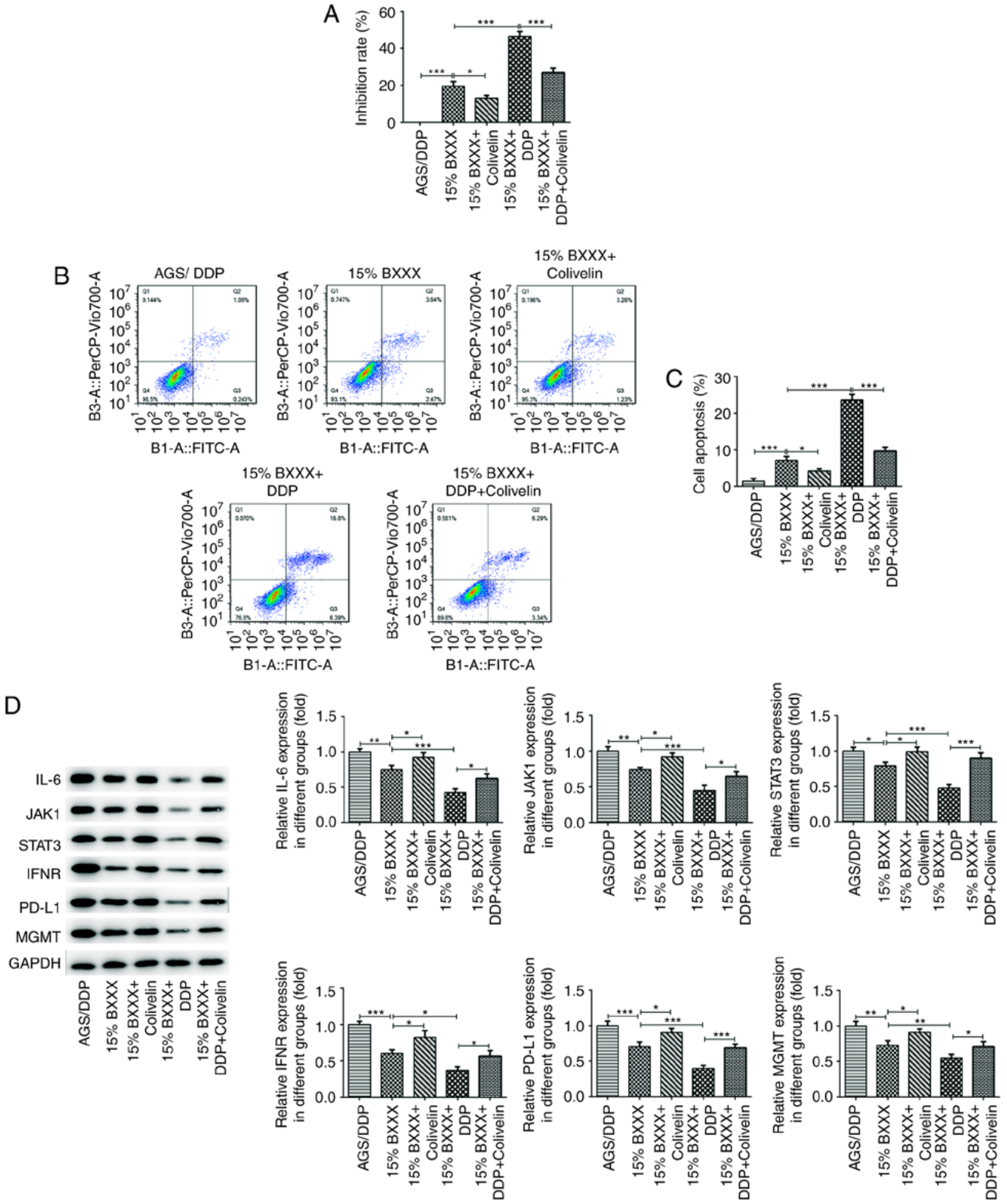

Figure 6. BXXX mediates drug-resistant GC cell sensitivity by regulating the expression of MGMT via IL-6/JAK/STAT3-mediated PD-L1. (A) A Cell Counting Kit-8 assay detected the viability in AGS/DDP cells. (B) Flow cytometry was used to detect cell apoptosis. (C) Statistical analysis of apoptotic cells. (D) Western blotting detected the expression levels of the signaling pathway-related proteins IL-6, JAK1, STAT3, IFNR and MGMT. "P $<0.05,{ }^{* *} \mathrm{P}<0.01$, ${ }^{* * * *} \mathrm{P}<0.001$. PD-L1, programmed cell death 1 ligand 1; MGMT, 6-O-methylguanine-DNA methyltransferase; DDP, cisplatin; BXXX, Banxia xiexin decoction; IFNR, IFN receptor.

of DDP-resistant lung cancer cells via the JAK/STAT3/PD-L1 pathways (31). Additionally, AP-2 was found to regulate the IL-6/JAK/STAT3 pathway and affect the expression of PD-L1, thus regulating drug resistance in glioma cells (22). Currently, the majority of PD-L1 studies focus on mechanisms of resistance to tumor immune escape by targeting the expression of PD-1 itself. PD-L1 can increase the expression of multidrug-resistant proteins, and is involved in the DNA damage and repair mechanisms of tumor cells (32-34). These findings indicate that PD-L1 expression is associated with that of MGMT. In the present study, an interaction between PD-L1 and MGMT was identified, and BXXX was found to mediate the expression of MGMT by regulating that of PD-L1 in drug-resistant GC cells. However, the current experiment did not further verify 

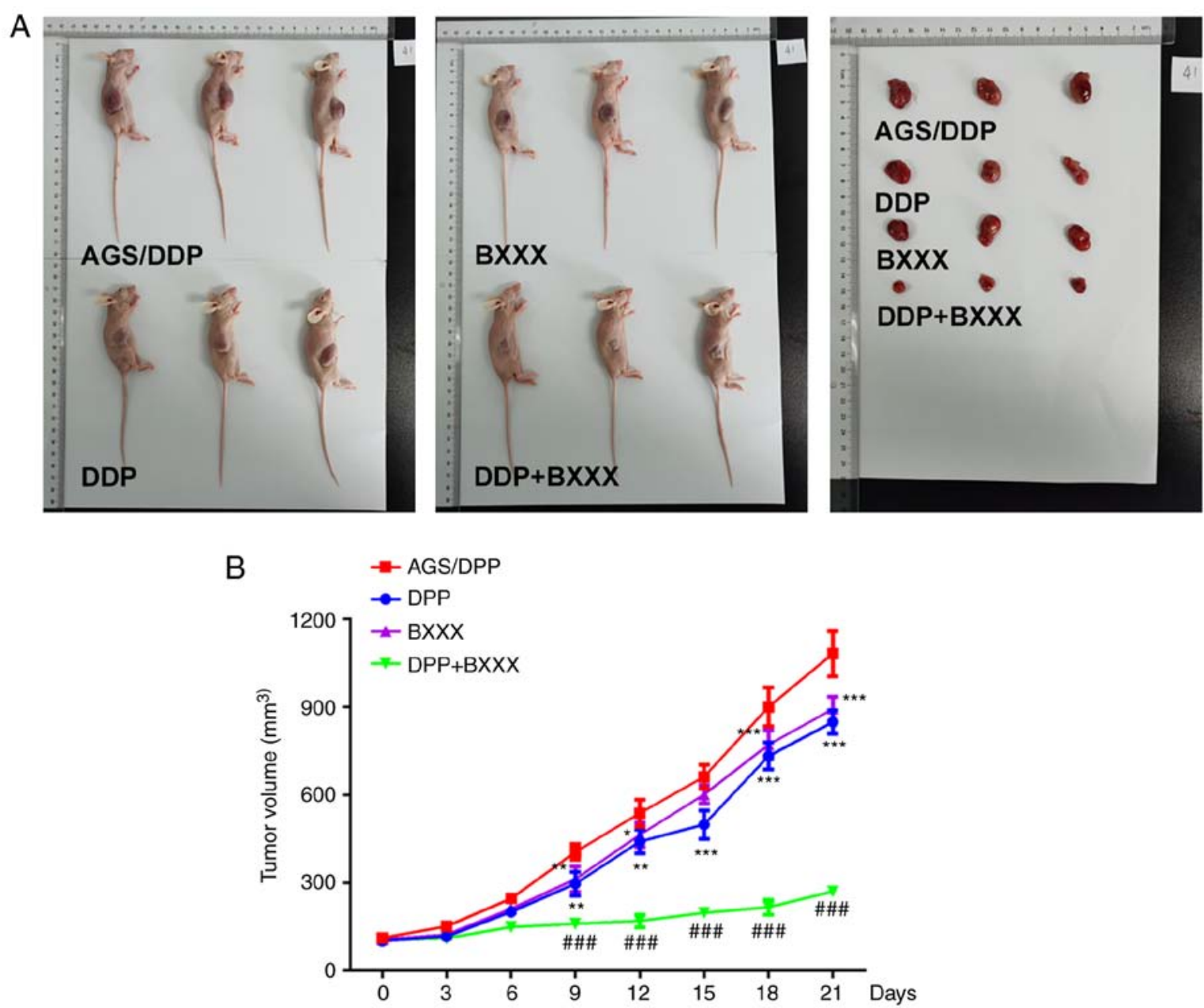

Figure 7. Tumor volume in a drug-resistant tumor-bearing mouse model of GC. (A) Images of tumor-bearing mice and tumor tissue. (B) Tumor volume statistics of different mice. ${ }^{*} \mathrm{P}<0.05,{ }^{* *} \mathrm{P}<0.01,{ }^{* * *} \mathrm{P}<0.001$ vs. AGS/DDP; ${ }^{\# \#} \mathrm{P}<0.01$ vs. DDP. DDP, cisplatin; BXXX, Banxia xiexin decoction.
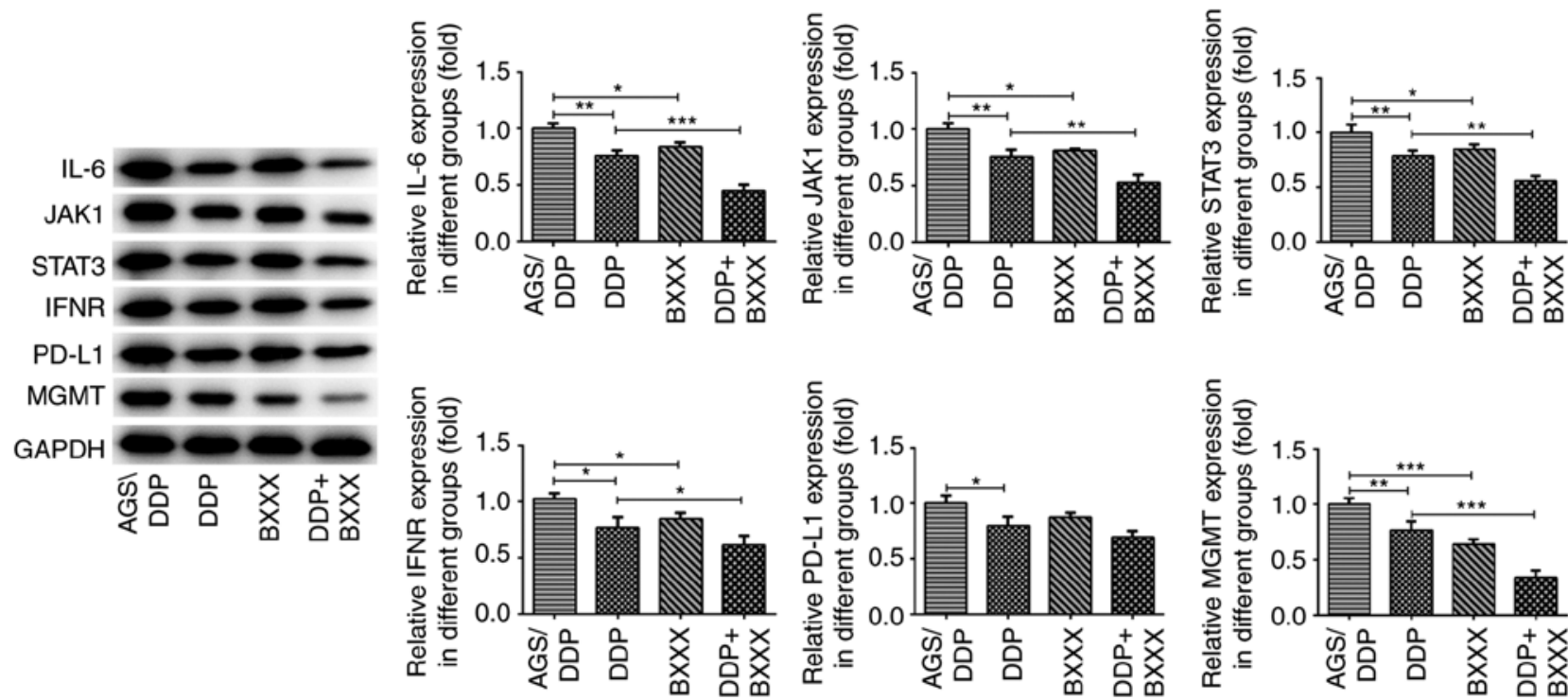

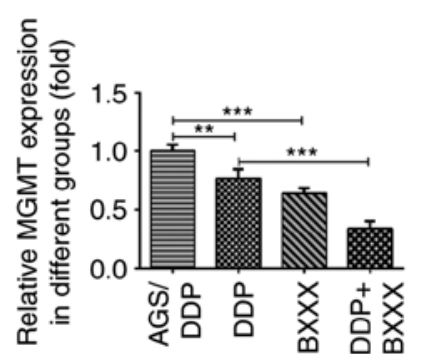

Figure 8. Expression levels of pathway-related proteins in the tumor tissue of tumor-bearing mice. ${ }^{*} \mathrm{P}<0.05,{ }^{* *} \mathrm{P}<0.01,{ }^{* * *} \mathrm{P}<0.001$. PD-L1, programmed cell death 1 ligand 1; MGMT, 6-O-methylguanine-DNA methyltransferase; DDP, cisplatin; BXXX, Banxia xiexin decoction; IFNR, IFN receptor.

the specific region of interaction between PD-L1 and MGMT, which may be a potential limitation of the present study. In future experiments, the binding region between PD-L1 and
MGMT were be further detected via relevant experiments. In the present study, colivelin (a STAT3 activator) reversed the inhibitory effects of BXXX on drug-resistant GC cells, as well 
as its repressive effect on PD-L1, indicating that BXXX had a direct effect on the pathways upstream of IFN $\gamma$ and IL-6, as well as on MGMT protein expression, but not on PD-L1 itself. Therefore, BXXX may influence the expression of PD-L1 by regulating the STAT3 pathway.

In the present study, AGS cells with high expression levels of PD-L1, STAT3 and MGMT, and NCL-N87 cells with no notable expression, were selected for drug resistance induction. The results suggested that the expression levels of PD-L1, MGMT and STAT3 were significantly increased, which was consistent with the two drug-resistant cell lines. This phenomenon reflects the effects of key pathway proteins (PD-L1, MGMT and STAT3) on the drug resistance and sensitivity of GC cells.

In conclusion, the results of the present study indicated that BXXX influenced the drug sensitivity of GC cells by regulating the expression of MGMT. This process was executed via the non-PD-1 targeting of PD-L1, which is mediate by IL-6/JAK/STAT3 signaling.

\section{Acknowledgements}

Not applicable.

\section{Funding}

This study was funded by the Research and Development Project in key areas of Guangdong Province (Study on the evolution law of microenvironment syndromes of stomach 'inflammation-cancer' and the combination of diseases and syndromes of strengthening; grant no. 163-2018-XMZC-0001165-0272).

\section{Availability of data and materials}

The datasets used and/or analyzed generated during the current study are available from the corresponding author on reasonable request.

\section{Authors' contributions}

XF wrote the manuscript and analyzed the data. QN and SH carried out the experiments. FX and GH made substantial contributions to analysis and interpretation of data, supervised the present study, searched the literature and revised the manuscript. All authors read and approved the final manuscript. QN and SH confirm the authenticity of all the raw data.

\section{Ethics approval and consent to participate}

The study protocol was approved by Affiliated Hospital of Yangzhou University, and all patients provided written informed consent. All of the procedures were in compliance with The Declaration of Helsinki and relevant policies in China. All animal experiments comply with the ethical requirements of the animal council.

\section{Patient consent for publication}

Not applicable.

\section{Competing interests}

The authors declare that they have no competing interests.

\section{References}

1. Rahman R, Asombang AW and Ibdah JA: Characteristics of gastric cancer in Asia. World J Gastroenterol 20: 4483-4490, 2014.

2. Bray F, Ferlay J, Soerjomataram I, Siegel RL, Torre LA and Jemal A: Global cancer statistics 2018: GLOBOCAN estimates of incidence and mortality worldwide for 36 cancers in 185 countries. CA Cancer J Clin 68: 394-424, 2018.

3. Choi AH, Kim J and Chao J: Perioperative chemotherapy for resectable gastric cancer: MAGIC and beyond. World J Gastroenterol 21: 7343-8, 2015.

4. Kawakami R, Mashima T, Kawata N, Kumagai K, Migita T, Sano T, Mizunuma N, Yamaguchi K and Seimiya H: ALDH1A3mTOR axis as a therapeutic target for anticancer drug-tolerant persister cells in gastric cancer. Cancer Sci 111: 962-973, 2020.

5. Liu X, Li M, Wang X, Dang Z, Yu L, Wang X, Jiang Y and Yang Z: Effects of adjuvant traditional Chinese medicine therapy on long-term survival in patients with hepatocellular carcinoma. Phytomedicine 62: 152930, 2019

6. Wang Z, Qi F, Cui Y, Zhao L, Sun X, Tang W and Cai P: An update on Chinese herbal medicines as adjuvant treatment of anticancer therapeutics. Biosci Trends 12: 220-239, 2018.

7. Yan S, Yue Y, Wang J, Li W, Sun M, Zeng L and Wang X: Banxia Xiexin decoction, a traditional Chinese medicine, alleviates colon cancer in nude mice. Ann Transl Med 7: 375, 2019.

8. Li K, Xu G, Liu C, Zhu B, Liu R, Hua B, Zhang W and Feng X: Effect of a modified Banxia Xiexin decoction plus chemotherapy on stage colon cancer. J Tradit Chin Med 39: 251-257, 2019.

9. Kim HR, Lee GS, Kim MS, Ryu DG, So HS, Moon HC, Lee YR, Yang SH and Kwon KB: Effects of Banxia Xiexin Decoction () on cisplatin-induced apoptosis of human A549 lung cancer cells. Chin J Integr Med 24: 436-441, 2018.

10. Su L, Wang MM, Xu MR, Wang X, Xia HZ, Zhang M, Zheng L, Zhu YD, Wang MQ and Li P: Banxia Xiexin Decoction () Combined with Afatinib in treatment of advanced gallbladder cancer: Case report and literature review. Chin J Integr Med 25: 303-306, 2019.

11. Liu XP, Ll PQ, Ming HX, Zhang W, Wang Q, Chen YW and Yang BL: Effects of Banxia Xiexin Decoction containing serum on proliferation, invasion and metastasis of gastric cancer peritoneal metastasis cell line GC9811-P. Zhongguo Zhong Xi Yi Jie He Za Zhi 36: 1224-1228, 2016 (In Chinese).

12. Yu Y, Zhang G, Han T and Huang H: Analysis of the pharmacological mechanism of Banxia Xiexin decoction in treating depression and ulcerative colitis based on a biological network module. BMC Complement Med Ther 20: 199, 2020.

13. Yang M, Chen J, Xu L, Shi X, Zhou X, An R and Wang X: A network pharmacology approach to uncover the molecular mechanisms of herbal formula Ban-Xia-Xie-Xin-Tang. Evid Based Complement Alternat Med 2018: 4050714, 2018.

14. Li C, Deng L, Shen H, Meng Q, Qian A, Sang H, Xia J and Li X: O-6-methylguanine-DNA methyltransferase inhibits gastric carcinoma cell migration and invasion by downregulation of matrix metalloproteinase 2. Anticancer Agents Med Chem 16: 1125-1132, 2016.

15. Nowicki TS, Hu-Lieskovan S and Ribas A: Mechanisms of resistance to PD-1 and PD-L1 blockade. Cancer J 24: 47-53, 2018.

16. Chen L, Diao L, Yang Y, Yi X, Rodriguez BL, Li Y, Villalobos PA, Cascone T, Liu X, Tan L, et al: CD38-mediated immunosuppression as a mechanism of tumor cell escape from PD-1/PD-L1 blockade. Cancer Discov 8: 1156-1175, 2018.

17. Pitt JM, Vétizou M, Daillère R, Roberti MP, Yamazaki T, Routy B, Lepage P, Boneca IG, Chamaillard M, Kroemer G and Zitvogel L: Resistance mechanisms to immune-checkpoint blockade in cancer: Tumor-intrinsic and -extrinsic factors. Immunity 44: 1255-1269, 2016.

18. Zhang Y, Xiang C, Wang Y, Duan Y, Liu C and Zhang Y: $\mathrm{PD}-\mathrm{L} 1$ promoter methylation mediates the resistance response to anti-PD-1 therapy in NSCLC patients with EGFR-TKI resistance. Oncotarget 8: 101535-101544, 2017. 
19. Zuo Y, Zheng W, Liu J, Tang Q, Wang SS and Yang XS: MiR-34a-5p/PD-L1 axis regulates cisplatin chemoresistance of ovarian cancer cells. Neoplasma 67: 93-101, 2020.

20. Gong K, Gong ZJ, Lu PX, Ni XL, Shen S, Liu H, Wang JW, Zhang DX, Liu HB and Suo T: PLAC8 overexpression correlates with PD-L1 upregulation and acquired resistance to chemotherapies in gallbladder carcinoma. Biochem Biophys Res Commun 516: 983-990, 2019.

21. Zhang X, Zeng Y, Qu Q, Zhu J, Liu Z, Ning W, Zeng H, Zhang N, Du W, Chen C and Huang JA: PD-L1 induced by IFN-gamma from tumor-associated macrophages via the JAK/STAT3 and $\mathrm{PI} 3 \mathrm{~K} / \mathrm{AKT}$ signaling pathways promoted progression of lung cancer. Int J Clin Oncol 22: 1026-1033, 2017.

22. Huang W, Zhong Z, Luo C, Xiao Y, Li L, Zhang X, Yang L, Xiao K, Ning Y, Chen L, et al: The miR-26a/AP-2alpha/Nanog signaling axis mediates stem cell self-renewal and temozolomide resistance in glioma. Theranostics 9: 5497-5516, 2019.

23. Chen G, Yang Y, Liu M, Teng Z, Ye J, Xu Y, Cai X, Cheng X, Yang $\mathrm{J}, \mathrm{Hu} \mathrm{C}$, et al: Banxia xiexin decoction protects against dextran sulfate sodium-induced chronic ulcerative colitis in mice. J Ethnopharmacol 166: 149-156, 2015.

24. Percie du Sert N, Ahluwalia A, Alam S, Avey MT, Baker M, Browne WJ, Clark A, Cuthill IC, Dirnagl U, Emerson M, et al: Reporting animal research: Explanation and elaboration for the ARRIVE guidelines 2.0. PLoS Biol 18: e3000411, 2020.

25. Livak KJ and Schmittgen TD: Analysis of relative gene expression data using real-time quantitative PCR and the 2(-Delta Delta C(T)) method. Methods 25: 402-408, 2001

26. Xiang Y, Guo Z, Zhu P, Chen J and Huang Y: Traditional Chinese medicine as a cancer treatment: Modern perspectives of ancient but advanced science. Cancer Med 8: 1958-1975, 2019.

27. Vasan N, Baselga J and Hyman DM: A view on drug resistance in cancer. Nature 575: 299-309, 2019.
28. Amatu A, Barault L, Moutinho C, Cassingena A, Bencardino K, Ghezzi S, Palmeri L, Bonazzina E, Tosi F, Ricotta R, et al: Tumor MGMT promoter hypermethylation changes over time limit temozolomide efficacy in a phase II trial for metastatic colorectal cancer. Ann Oncol 27: 1062-1067, 2016.

29. Li Y, Yang Y, Lu Y, Herman JG, Brock MV, Zhao P and Guo M Predictive value of CHFR and MLH1 methylation in human gastric cancer. Gastric Cancer 18: 280-287, 2015.

30. Lei Y, Tang L, Hu J, Wang S, Liu Y, Yang M, Zhang J and Tang B: Inhibition of MGMT-mediated autophagy suppression decreases cisplatin chemosensitivity in gastric cancer. Biomed Pharmacother 125: 109896, 2020.

31. Shen M, Xu Z, Xu W, Jiang K, Zhang F, Ding Q, Xu Z and Chen Y: Inhibition of ATM reverses EMT and decreases metastatic potential of cisplatin-resistant lung cancer cells through JAK/STAT3/PD-L1 pathway. J Exp Clin Cancer Res 38: 149, 2019.

32. Cha JH, Chan LC, Li CW, Hsu JL and Hung MC: Mechanisms controlling PD-L1 expression in cancer. Mol Cell 76: 359-370, 2019.

33. Jiang X, Wang J, Deng X, Xiong F, Ge J, Xiang B, Wu X, Ma J, Zhou M, Li X, et al: Role of the tumor microenvironment in PD-L1/PD-1-mediated tumor immune escape. Mol Cancer 18: $10,2019$.

34. Kim JM and Chen DS: Immune escape to PD-L1/PD-1 blockade: Seven steps to success (or failure). Ann Oncol 27: 1492-504, 2016.

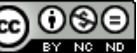

This work is licensed under a Creative Commons Attribution-NonCommercial-NoDerivatives 4.0 International (CC BY-NC-ND 4.0) License. 